\title{
Cytokine profile of patient's sera of Leptospirosis to OMP of Leptospira interrogans serovar tarassovi
}

\author{
Prem Latha $\mathrm{MM}^{1}$, Kaur IR ${ }^{2}$, Avasthi $\mathbf{R}^{3}$, Dey $\mathrm{AB}^{4}$, Chaudhry $\mathbf{R}^{5}$ \\ ${ }^{1}$ Amity Institute of Virology and Immunology, Amity University Noida, Uttar Pradesh, ${ }^{2}$ Department of Microbiology, University College of Medical \\ Sciences Guru Tegh Bahadur Hospital, Delhi, ${ }^{3}$ Department of Medicine, University College of Medical Sciences Guru Tegh Bahadur Hospital, \\ Delhi, ${ }^{4}$ Department of Medicine, All India Institute of Medical Sciences, New Delhi, ${ }^{5}$ Department of Microbiology, All India Institute of Medical \\ Sciences, New Delhi
}

\section{A B S T R A C T}

Aims and Objectives: In the past decade, Leptospirosis has emerged as a globally important emerging infectious disease. Leptospires have several chemical components such as Lipoproteins, glycolipoproteins which are toxic and participate in the pathogenesis of the disease. This study was focused to find out the immune response of outer membrane proteins (OMPs) of Leptospira interrogans serovar tarassovi by estimating the cytokine profile and antibody levels from cases of Leptospirosis. Materials and Methods: A total of 120 blood samples were collected from patients (90 male/30 female) presenting with acute febrile illness and fulfilling the criteria of clinical diagnosis of Leptospirosis. All the 120 samples \& 40 healthy controls were subjected to ELISA (IgM serion), MAT and Leptospira interrogans serovar tarassovi IgM ELISA and also to detect TNF $\alpha$ and IL-6 levels using (BD Opt EIA Human IL-6 \& TNF $\alpha$ ELISA kits). Results: Sera which were found to be reactive in L. interrogans serovar tarassovi OMP IgM ELISA had significant levels of TNF $\alpha$ and IL- 6 and antibody titres in ELISA and MAT. Conclusions: Patients from Group I had significant levels of TNF $\alpha$ in the serum i.e., $p=0.02$ which indicates the pro-inflammatory Th1 response as TNF $\alpha$ are macrophage derived cytokine mediators. The IL-6 level of Group I, II, III were significantly higher than the Group IV ( $p=0.02$ ) suggesting that Th2 anti-inflammatory response seen in majority of these cases which has a protective role in leptospirosis.

Key words: OMPs, Leptospira, TNF $\alpha$ and IL-6

\section{INTRODUCTION}

Leptospirosis is an acute bacterial disease caused by the genus Leptospira. Members of the Leptospira are grouped into serovars on the basis of their antigenic relatedness where more than 260 serovars have been recognized. The Leptospira cytoplasmic membrane is closely associated with a peptidoglycan cell-wall, which is overlaid by an outer-membrane, phospholipid, outer-membrane proteins (OMPs). Several Leptospiral OMPs have been shown to attach the host extracellular matrix which are responsible for adhesion of Leptospira to host tissue.

This study has been undertaken to find the immune reaction of the OMPs to the patient's sera of Leptospirosis by estimating the cytokine profile and antibody levels.

\section{MATERIALS AND METHODS}

A total of 120 blood samples were collected from patients (90 male/30 female) with febrile illness and fulfilling the criteria of clinical diagnosis of Leptospirosis who were admitted to Medical wards of University College of Medical Sciences, GTBH, Delhi and All India Institute of Medical Sciences, Hospital, New Delhi. These samples were collected during the period from October 2002 August 2005. Serum was separated and stored at $-20^{\circ} \mathrm{C}$ and 40 healthy controls were also collected with no history of fever.

- The total samples were categorized into the following groups by fulfilling different criteria and methods as: Criteria for confirmatory diagnosis: (Group I) Demonstration of 1:200 titers against different sero- 
groups in microscopic agglutination test

- Criteria for presumptive diagnosis: (Group II) A suspected case of Leptospirosis positive by MAT $(1: 100)$

- Clinically suspected case of leptospirosis but serologically negative (ELISA, MAT and Dridot) (Group - III)

- Healthy Controls: (Group IV).

IgM ELISA: Performed according to the manufacturer`s instructions. Evaluation of the test was done by serion ELISA classic Leptospira (Institute virion, serion $\mathrm{GmbH}$, Wurzhung, Germany).

Microscopic agglutination test (MAT): Performed using Faine's methodology ${ }^{1}$ according to WHO guidelines.

L. tarassovi OMP IgM ELISA: Performed according to the method of Premlatha et al. ${ }^{2}$

Estimation of IL-6 and TNF $\alpha$ : Using (BD Opt EIA ${ }^{\text {TM }}$ HUMAN IL-6, TNF $\alpha$ ELISA kits) was performed according to manufacturer instructions.

\section{RESULTS}

The level of TNF $\alpha$ and IL-6 were estimated from the four different Groups each having 40 samples. The patients from Group I had significant levels of TNF $\alpha$ in the serum (i.e., $p=0.02)$. The mean of IL-6 level of Group I and Group II were significantly higher than the Group III. Sera which were found to be reactive in L.tarassovi OMP IgM ELISA, had showed significant levels of TNF $\alpha$ and IL-6 and had antibody titre in IgM ELISA and MAT.

\section{DISCUSSION}

Pathogenic Leptospira causes Intercellular infection. The immunological reaction of host to intercellular infection is unique. The potential immunogen of expressed protein

\begin{tabular}{lcccc}
\multicolumn{5}{l}{$\begin{array}{l}\text { Table 1: Profile of cytokine and antibody } \\
\text { production during human }\end{array}$} \\
$\begin{array}{l}\text { L. tarassovi } \\
\text { OMP IgM ELISA }\end{array}$ & TNFa & IL-6 & $\begin{array}{c}\text { ELISA } \\
\text { (IgM) }\end{array}$ & MAT \\
\hline $\begin{array}{l}\text { Group I } \\
29 / 40\end{array}$ & 27 & 24 & 20 & 27 \\
$\begin{array}{l}\text { Group II } \\
19 / 40\end{array}$ & 9 & 12 & 10 & 10 \\
$\begin{array}{l}\text { Group III } \\
8 / 40 \\
\text { Healthy control } \\
40\end{array}$ & - & - & - & - \\
\hline
\end{tabular}

should be degraded by antigen presenting cells in the hosts, and should have antigenic epitope which recognizes MHC II protein and $\mathrm{T}$-cell receptor.

A total of 120 serum samples and 40 healthy controls were subjected to L. tarassovi OMP IgM ELISA, IgM ELISA and MAT, and estimation of IL- 6 and $\mathrm{TNF} \alpha$ was done, using (BD Opt EIA ${ }^{\mathrm{TM}}$ HUMAN IL-6, TNF $\alpha$ ELISA kits).

In this study the patients from Group I had significant levels of $\mathrm{TNF} \alpha$ in their serum (i.e., $\mathrm{p}=0.02$ ) which indicates pro-inflammatory $\mathrm{Th}_{1}$ response as $\mathrm{TNF} \alpha$ are macrophage derived cytokine mediators. Most of these patients had high morbidity. In a study Tajiki and Salomao circulating level of $\mathrm{TNF} \alpha$ has been detected in patients with Leptospriosis which associated with severity of the disease. ${ }^{3}$

The mean of IL-6 level of Group I and Group II were significantly higher than the Group VI $p=0.02$ suggesting that $\mathrm{Th}_{2}$ anti inflammatory response seen in majority of these cases.

Studies by Dorigatti et $\mathrm{al}^{4}$ has shown that OMP of pathogenic Leptospira when inoculated to tubular cells in culture activated nuclear NF Kappa B binding and stimulated downstream inducible nitric oxide (INOS), monocyte chemoattractant protein-1 (MOP-1) and tumor necrosis factor. Another study reported by Magrinho et $\mathrm{al}^{5}$ detected TNF $\alpha$ and IL-6 in rat renal experimentally infected with L.canicola.

In this study sera which were found to be reactive with $\mathrm{L}$. tarassovi OMP had significant levels of TNF $\alpha$ and IL-6 and also reacted positive in MAT and ELISA (Table 1). The detection of TNF $\alpha$ and IL-6 in the sera suggests that OMPs play an important role in the interaction of host cells with Leptospira during infection.

\section{CONCLUSION}

Leptospires enter the body through small cuts, via mucous membranes such as the conjunctiva or skin. They circulate in the blood stream, with the bacteremic phase lasting for up to 7 days The second stage of acute leptospirosis is immune phase, with the disappearance of the organism from the bloodstream and the appearance of antibodies .It was observed that the extracted OMP of L.tarassovi reacted to the patient sera to produce cytokines i,e TNF alpha and IL-6 and also produced antibodies during the course of Infection. Proteins play a major role in binding to various components of the extra cellular matrix and thereby mediate host pathogen interaction and the proteins which are expressed during infection play an important role in immuno-pathogenesis. 


\section{REFERENCES}

1. Faine S. Guidelines for the control of Leptospirosis WHO offset publication 1982; (67). World Health Organization, Geneva.

2. PremLatha MM, Kaur IR, Avasthi R, Dey AB and Chaudhry R. A newer approach for the sero-diagnosis of leptospirosis using outer membrane proteins of Leptospira interrogans serovar Tarassovi, Asian Journal of Medical Sciences 2013; 4(2): 41-46.

3. Tajiki MH and Salomão R. Association of plasma levels of tumor necrosis factor $\alpha$ with severity of disease and mortality among patients with leptospirosis. Clin Infect Dis1996; 23: $1177-1178$.

4. Dorigatti F, Brunialti MKC, Romero EC, Kallas EG and Salomão F. Leptospira interrogans activation of peripheral blood monocyte glycolipoprotein demonstrated in whole blood by the release of IL-6. Brazilian Journal of Medical and Biological Research 2005; 38: $909-914$.

5. Marinho M, Monteiro CMR, Peiró JR, Machado GF and Oliveira Júnior IS. TNF- $\alpha$ and IL-6 immunohistochemistry in rat renal tissue experimentally infected with Leptospira interrogans serovar Canicola. J Venom Anim Tox incl Trop Dis 2008;14: 533-534.

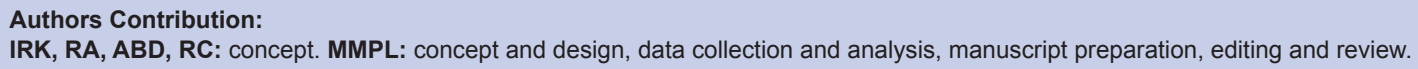

